\title{
Acquisition of Direct Sequence Spread Spectrum Acoustic Communication Signals
}

\author{
M.Stojanovic and L.Freitag* \\ Massachusetts Institute of Technology \\ Cambridge, MA 02139 \\ *Woods Hole Oceanographic Institution \\ Woods Hole, MA 02543
}

\begin{abstract}
Acquisition of the code timing in DS/SS systems is addressed for time-varying frequency-selective underwater acoustic channels. Acquisition algorithm based on adaptive channel estimation and fitlering is proposed. The algorithm employs feedback of pre-cursor and post-cursor inter-chip interference to provide a channel estimate from which the code timing is acquired. The channel estimate is also used to filter the signal prior to making the acquisition decision. Channel estimation / filtering procedure eliminates the need for explicit equalization, and enables a computationally efficient implementation. Simulation results show excellent performance in low-SNR regime, as well as in the presence of multiple-access interference and fast fading.
\end{abstract}

Index Terms - Underwater acoustic communications, codedivision multiple access, spread spectrum, code acquisition, adaptive MMSE receiver, channel estimation, equalization, tap selection, phase synchronization.

\section{INTRODUCTION}

Spread spectrum signaling provides a basis for two types of underwater acoustic communication systems: those that require low probability of intercept (LPI) and those that accommodate multiple users based on code-division multiple-access (CDMA). Spread spectrum methods are distinguished as either frequency-hopping (FH/SS) or direct-sequence (DS/SS) methods, and a comparative analysis of the performance of these two methods on acoustic underwater communication channels was presented in [1]. While FH/SS offers simplicity of implementation through noncoherent detection of FSK modulated signals, DS/SS offers improved performance on time-varying multipath channels through coherent detection of high-resolution PSK modulated spreading codes. In addition, it provides signals whose level can be kept below that of the noise, thus providing an LPI capability that is better suited for certain applications. From the viewpoint of multiuser communications, spread spectrum techniques offer a soft capacity limit that is not available with either the time-division or frequency-division multipleaccess schemes.

A DS/SS receiver uses knowledge of the spreading code to detect the received signal that is buried in noise or contaminated by interference from other users of the system. In order to apply the known code to signal despreading and detection, the system must first be synchronized. Synchronization process occurs in two phases: acquisition and tracking. During the acquisition phase, the received signal is coarsely aligned in time with the locally generated replica of the spreading code. Once the initial alignment has been performed, the actual data detection can begin. To ensure proper signal processing during this phase, fine synchronization, or tracking, must be maintained.

In applications that require low probability of intercept, initial synchronization must be acquired without the aid of special signal preambles. In other words, acquisition must be performed directly on the DS/SS signal, which, for the time allocated to acquisition, is modulated using an all-ones data stream. This is not necessary in multiuser applications; however, it is sometimes desired that acquisition be performed without the aid of out-of-band synchronization signals. Typically, acquisition can be performed in two ways: through a parallel or a serial search [2], [3]. In a parallel search, the received signal is simultaneously despread using all the possible shifts of the spreading code. The despreader, or the correlator, that produces the largest output corresponds to the correct sequence timing. The number of correlators needed is proportional to the length of the spreading sequence. Serial acquisition is a method by which correlation for despreading starts with an arbitrarily chosen sequence shift, and progresses for a pre-determined interval of time after which a decision can be made as to whether the assumed sequence shift was correct or not. If the correlator output exceeds a threshold, acquisition is declared successful; otherwise, the locally generated sequence is shifted, and a new shift tested in the same manner. The receiver complexity is thus reduced at the expense of an increased time needed for acquisition.

The most rudimentary detection methods are based on the use of a correlator only, and are suited for channels that have no multipath. If the channel introduces multipath, a correlator will produce an ambiguous output. To prevent false acquisition in such a situation, the correlator must be preceeded by an adaptive filter or combined with it. Such an approach was investigated for wireless radio channels in [4]. In this refer- 
ence, a simple receiver was proposed based on LMS adaptive filtering and a serial acquisition search. For each assumed sequence shift, the receiver is run for a pre-specified interval of time using a known training sequence of data symbols. The filter output is despread using the known sequence with an assumed starting time. The error between the so-obtained data symbol estimate and the known training data is used to update the filter coefficients at the symbol rate. At the end of the allotted time interval, the mean squared error is checked for convergence. If it is decided that convergence exists, the filter tap weights are used to determine the exact sequence timing. This action is performed by finding the point of maximal correlation between the filter weights and the spreading sequence used. If it is established that there is no convergence, the sequence is shifted by a certain number of chips, and the process is repeated.

Similar techniques based on adaptive minimum mean squared error (MMSE) filtering for timing acquisition were also investigated for DS/SS CDMA applications [5], [6]. In multiuser systems, the major problem is detection of the desired user's signal in the presence of multiple-access interference. Whether because the system is asynchronous, or there exists multipath propagation, the signals arriving from different users do not appear orthogonal at the receiver, and, thus, a simple decorrelator does not suffice to suppress the interference. Instead, an adaptive MMSE receiver is often employed. Acquiring synchronization in such a situation is based on applying the adaptive MMSE receiver for each supposed initial timing. While [5] addresses a parallel symbol-timing acquisition scheme for systems that use short spreading sequences with one period per symbol, [6] treats a serial acquisition scheme, somewhat similar to that of [4]. Namely, an adaptive receiver is employed, but without explicit despreading. Instead, it is left to the adaptive filter to perform both despreading and interference suppression. Such an approach can be justified for spreading sequences of short period that allow use of manageable size adaptive filters. After convergence has been established, the filter tap-weights are cross-correlated with the spreading code to obtain the desired sequence timing. It was found that fast convergence of RLS algorithm makes it preferable to the LMS algorithm. However, application of these techniques remains limited to systems with short spreading codes and channels with slow time-variation.

An underwater acoustic channel is often characterized by extended multipath propagation and rapid temporal variation. The benefits of DS/SS signaling in such a situation are conditioned on the receiver's ability to track the time-variation of the channel. Time-variation occurs in an underwater acoustic channel because of the natural variations in the medium, and because of the transmitter/receiver motion. The motioninduced Doppler distortion is the most prominent factor contributing to the channel variability in mobile underwater systems that employ autonomous underwater vehicles (AUVs) equipped with acoustic modems.
The implications of time-varying underwater multipath on the design of a DS/SS system are twofold. First, multipath dispersion causes interference between adjacent chips that can often extend over tens of chip intervals, and second, channel variation may be significant over the duration of a single data symbol. Neither of these effects is present in wireless radio systems. To account for the degradation in system performance, a dedicated receiver design is necessary for acoustic systems. In general, multipath degradation can be treated by adaptive equalization using chip-spaced decision-feedback filters. For slowly varying channels, symbol-rate adaptation is sufficient [7], while for more rapidly varying channels, adaptation must be carried out at the chip rate [8]. DS/SS receivers proposed in [7] and [8] focus on signal processing aspects of multipath equalization and interference suppression, relying on side techniques to initially acquire synchronization. Usually, a probe signal, based on high-resolution pseudo-random code or a frequency sweep is used to determine the starting time of a data packet. In this paper, we focus on the aspects of acquisition, and propose a method suitable for use with any DS/SS receiver in an underwater acoustic environment characterized by time-varying multipath.

The proposed method capitalizes on the fact that acquisition process is equivalent to that of channel estimation. Acquisition methods [4]-[6] for time-varying multipath channels rely on observing the tap coefficients of an adaptive linear equalizer to infer the starting time of the spreading code. It is possible to do so because the equalizer coefficients are correlated with those of the channel. Rather than using an adaptive equalizer, the method proposed is based on channel estimation. In this method, a channel estimate is obtained explicitly, using a technique similar to that of [9]. The position of the channel tap with largest magnitude within the spreading code length indicates the starting time of the code. Hence, no inference needs to be made from the equalizer taps. At the same time, the channel estimate is used to compute the coefficients of a filter, which is used to equalize the signal prior to despreading. The acquisition decision is made from the observation of the error at the equalizer output. This error is also used to generate feedback for the phase-locked loop, which enables acquisition in the presence of an unknown Doppler shift. The advantage of this approach is that channel taps can be updated independently, and, hence, the acquisition time does not depend on the equalizer length and its convergence time, as it does in techniques based on direct equalization. In addition, because multipath is naturally sparse, it suffices to update only the selected channel taps.

The principles of acquisition algorithm are presented in Sec.II. The algorithm performance is tested in simulation, and results are presented in Sec.III. Performance is assessed both in the low SNR regime for the single-user case representative of an LPI application, and in the multiuser, CDMA case. Conclusions are summarized in Sec.IV. 


\section{ACQUISITION ALGORITHM}

The acquisition principles are based on modeling the complex baseband received signal as

$$
v(t)=\sum_{l} p(l) h\left(t-l T_{c}\right)+w(t)
$$

where $p(l)$ are the chips of the spreading sequence (there is no data modulation), $h(t)$ is the impulse response of the channel (including any transmit/receive filtering), $T_{c}$ is the chip duration, and $w(t)$ is the noise, which is assumed to be uncorrelated with the signal. The code period is chosen greater than the multipath spread, $L T_{c}>T_{m}$. For the time being, it is assumed that there is no frequency offset, and the problem of carrier synchronization is treated later.

The received signal is sampled at the Nyquist rate, taken without loss of generality to be $2 / T_{c}$ for the signal band-limited to $\pm 1 / T_{c}$. The signal samples are arranged in a vector

$$
\mathbf{v}(k)=\sum_{l} \mathbf{h}(l) p(k-l)+\mathbf{w}(k)
$$

where the time span of the vectors is equal to the spreading code duration, $L T_{c}$. All the vectors are defined as column vectors, and time-indexed such that

$$
\mathbf{v}(k)=\left[\begin{array}{c}
v\left((k+L-1) T_{c}+T_{c} / 2\right) \\
v\left((k+L-1) T_{c}\right) \\
\vdots \\
v\left(k T_{c}+T_{c} / 2\right) \\
v\left(k T_{c}\right)
\end{array}\right]
$$

Note that the channel vector

$$
\mathbf{h}(l)=\left[\begin{array}{c}
h\left((l+L-1) T_{c}+T_{c} / 2\right) \\
h\left((l+L-1) T_{c}\right) \\
\vdots \\
h\left(l T_{c}+T_{c} / 2\right) \\
h\left(l T_{c}\right)
\end{array}\right]
$$

is related to $\mathbf{h}(l-1)$ through a shift-down-by-two operation (or as many as there are samples per chip). There is a total of $2 L+1$ non-zero shifts. The channel vector $\mathbf{h}(0)$ is taken to be the reference vector (with respect to the chip sequence $p(k)$ ).

When the sequence $p(k)$ is known, a channel estimation algorithm is based on the fact that the noise is uncorrelated with the signal, and, hence,

$$
\mathbf{h}(0)=E\left\{\mathbf{v}(k) p^{*}(k)\right\}
$$

From this expression, a simple adaptive channel-estimation algorithm can be obtained [9]:

$$
\hat{\mathbf{h}}[k]=\lambda \hat{\mathbf{h}}[k-1]+(1-\lambda) \mathbf{v}(k) p^{*}(k)
$$

In this algorithm, $\hat{\mathbf{h}}[k]$ denotes the estimate of the reference vector $\mathbf{h}(0)$ obtained in the $k$ th iteration, i.e., $\hat{\mathbf{h}}[k]=\hat{\mathbf{h}}(0, k)$, and $\lambda<1$ is the exponential filtering factor. This algorithm was successfully used in channel-estimation-based equalization of high-rate underwater acoustic signals.

Once a channel estimate is available, its coefficients indicate the relative delays of the channel paths. The coefficient with largest magnitude can thus be used to determine the code timing. Namely, if

$$
m=\arg \max \mathbf{h}(0)=\arg \max \left\{h\left(i T_{c} / 2\right)\right\}_{i=0}^{2 L-1}
$$

then the delayed input siganl $v\left(k T_{c}+m T_{c} / 2\right)$ is timesynchronized with the code $p(k)$. Because the code is periodic, and the period is greater than the multipath spread, there is no ambiguity about the timing of the principal component.

If there is no multipath, the time-aligned signal is immediately despread using the sequence of known chips $p(k)$. In the presence of multipath, inter-chip interference must first be suppressed. This is accomplished by filtering, or equalization. The chip estimates obtained after equalization, $\hat{p}(k)$, are fed to the despreader. The quality of the chip estimates, and consequently, the acquisition performance, relies on two factors: (1) the quality of the channel estimate, and (2) the quality of the equalizer.

Since the channel estimate indicates the positions and strengths of multipath components, it can be used to compute the coefficients of an equalizer. For purposes of data detection, a procedure for computing the coefficients of a decisionfeedback equalizer is given in [9]. For purposes of acquisition, the data sequence is known (an all-one sequence is normally used). This fact may be exploited to obtain both an improved channel estimate and an equalization method superior to that of [9]. The channel estimation / filtering procedure is based on the feedback of both post-cursor and pre-cursor inter-chip interference terms, as described below.

\section{A. Channel estimation}

In the presence of multipath, the received signal can be represented as

$$
\mathbf{v}(k)=\mathbf{h}(0) p(k)+\sum_{l \neq 0} \mathbf{h}(l) p(k-l)+\mathbf{w}(k)
$$

From this expression, it follows that the signal

$$
\mathbf{v}_{0}(k)=\mathbf{v}(k)-\sum_{l \neq 0} \mathbf{h}(l) p(k-l)
$$

can be expressed as

$$
\mathbf{v}_{0}(k)=\mathbf{h}(0) p(k)+\mathbf{w}(k)
$$

This signal is free of multipath interference terms, and, hence, it can be used to obtain a better channel estimate. This signal also satisfies the condition

$$
\mathbf{h}(0)=E\left\{\mathbf{v}_{0}(k) p^{*}(k)\right\}
$$


The estimate of the channel can thus be obtained recursively as

$$
\hat{\mathbf{h}}[k]=\lambda \hat{\mathbf{h}}[k-1]+(1-\lambda) \mathbf{v}_{0}(k) p^{*}(k)
$$

Because the chip sequence is known, the signal $\mathbf{v}_{0}(k)$ can be generated from the input $\mathbf{v}(k)$ using the expression (9) with channel estimates instead of true values:

$$
\left.\hat{\mathbf{v}}_{0}(k)=\mathbf{v}(k)-\sum_{l \neq 0} \hat{\mathbf{h}}(l) p(k-l)\right)
$$

Channel estimates $\hat{\mathbf{h}}(l)$ are shifts of the reference vector $\hat{\mathbf{h}}(0, k)$, and this property can be exploited to compute (13) in an efficient manner (see Sec. II-E).

\section{B. Filtering (equalization)}

Once the channel estimate is available, it is used to filter the received signal in order to suppress the inter-chip interference. The filtering operation is ordinarily performed on the signal $\mathbf{v}(k)$, in which case the filter coefficients must be computed from the channel estimate. However, filtering can be performed directly on the signal $\mathbf{v}_{0}(k)$, given in (9). The MMSE filter for this signal is defined simply by the (scaled) channel vector $\mathbf{h}(0)$. Using the available channel estimates, the filtering operation is defined by

$$
\hat{p}(k)=\frac{1}{E_{0}(k)} \hat{\mathbf{h}}^{\prime}(0, k) \hat{\mathbf{v}}_{0}(k)
$$

where

$$
E_{0}(k)=\hat{\mathbf{h}}^{\prime}(0, k) \hat{\mathbf{h}}(0, k)
$$

\section{Acquisition test}

Equations (12), (13), and (14) define the signal processing needed before the acquisition test is performed. The test leads to the decision on whether the correct sequence timing has been acquired. The simplest test consists of checking for consistency in the estimated timing. If it has not changed for a certain duration of time, acquisition is declared. However, there is nothing to guarantee the correctness of the so-obtained timing estimate. Also, such test is not appropriate for timevarying channels. To account for correctness, the acquisition test is performed by observing the despreader output. In the absence of data modulation, the quality of chip estimates can be monitored in a continuously running despreading operation:

$$
\hat{d}(k)=\frac{1}{N} \sum_{i=k-N+1}^{k} \hat{p}(i) p^{*}(i)
$$

where $N$ is the despreader length, and the quantity $\hat{d}(k)$ represents an estimate of the data symbol, which is +1 in the absence of data modulation. The resulting mean squared error, $E\left\{|1-\hat{d}(k)|^{2}\right\}$, can be used to determine the success of acquisition. Once this error falls below a pre-specified threshold,
$E_{\text {test }}$, acquisition is declared. Alternatively, symbol decisions can be made at the despreader output:

$$
\tilde{d}(k)=\operatorname{sgn}(\hat{d}(k))
$$

The acquisition test now consists of observing a pre-specified number, $N_{\text {test }}$, of correct $(+1)$ decisions.

\section{Carrier synchronization}

The procedure outlined above was obtained under the assumption that there exists carrier synchronization. If this is not so, the received signal is modeled as

$$
v_{\theta}(t)=\sum_{l} p(l) h\left(t-l T_{c}\right) e^{j \theta(t)}+w(t)
$$

Carrier synchronization is performed using an estimate of the carrier phase $\hat{\theta}(t)$ :

$$
v(t)=v_{\theta}(t) e^{-j \hat{\theta}(t)}
$$

In a digital receiver, operating at the chip rate, carrier phase estimate is obtained once per chip interval, $\hat{\theta}\left(k T_{c}\right)=\hat{\theta}(k)$. The phase-corrected signal is used as the input to acquisition algorithm.

Carrier phase estimation can be performed in a manner that minimizes the mean squared error in the chip estimation process. The chip estimation error is given by

$$
e_{p}(k)=p(k)-\hat{p}(k)
$$

where $\hat{p}(k)$ is expressed in terms of the carrier phase estimate as

$$
\hat{p}(k)=\frac{1}{E_{0}} \mathbf{h}^{\prime}(0)\left[\mathbf{v}_{\theta}(k) e^{-j \hat{\theta}}-\sum_{l \neq 0} \mathbf{h}(l) p(k-l)\right]
$$

The phase-error signal, used to drive the PLL can now be obtained as

$$
-\frac{\partial\left|e_{p}^{2}(k)\right|}{2 \partial \hat{\theta}}=\psi(k)=\operatorname{Im}\left\{\frac{1}{E_{0}} \mathbf{h}^{\prime}(0) \mathbf{v}(k) e_{p}^{*}(k)\right\}
$$

The PLL is implemented as a second order filter:

$$
\hat{\theta}(k+1)=\hat{\theta}(k)+K_{f 1} \hat{\psi}(k)+K_{f 2} \sum_{i \leq k} \hat{\psi}(i)
$$

where $\hat{\psi}(k)$ is evaluated from (21) using the channel estimates instead of the true values.

An alternative to the above carrier synchronization algorithm is one that uses the error after despreading instead of the chip error. The error after despreading is given by

$$
e_{d}(k)=1-\frac{1}{N} \sum_{k-N+1}^{k} p^{*}(i) \mathbf{h}^{\prime}(0)\left[\mathbf{v}_{\theta}(i) e^{-j \hat{\theta}}-\sum_{l \neq 0} \mathbf{h}(l) p(k-l)\right]
$$


The resulting phase-error signal is

$$
-\frac{\partial\left|e_{d}^{2}(k)\right|}{2 \partial \hat{\theta}}=\psi(k)=\operatorname{Im}\left\{\frac{1}{E_{0} N} \sum_{k-N+1}^{k} p^{*}(i) \mathbf{h}^{\prime}(0) \mathbf{v}(i) e_{d}^{*}(k)\right\}
$$

The PLL operation is still defined again by (22).

\section{E. Algorithm summary}

For ease of notation, the channel estimate at iteration $k$ is repesented as

$$
\hat{\mathbf{h}}[k]=\left[\begin{array}{c}
\underline{\hat{\mathbf{h}}}_{L-1}(k) \\
\vdots \\
\hat{\mathbf{h}}_{0}(k)
\end{array}\right]
$$

where the underlined quantities indicate 2-element vectors (the two samples correspond to one chip interval). The acquisition algorithm is defined by the following steps, carried out in every iteration $k$, corresponding to the $k$ th chip of the spreading sequence:

1) Take two new samples of the received signal and perform carrier synchronization:

$$
\underline{\mathbf{v}}(k+L-1)=\left[\begin{array}{c}
v\left((k+L-1) T_{c}+T_{c} / 2\right) \\
v\left((k+L-1) T_{c}\right)
\end{array}\right] e^{-j \hat{\theta}(k)}
$$

2) Form the signal vector:

$$
\mathbf{v}(k)=\left[\begin{array}{c}
\underline{\mathbf{v}}(k+L-1) \\
\downarrow \mathbf{v}(k-1)
\end{array}\right]
$$

Here, $\downarrow$ indicates taking all but the bottom two elements of a vector. (In general, as many elements as there are samples per chip are shifted out.)

3) Form the vector

$$
\hat{\overline{\mathbf{v}}}(k)=\left[\begin{array}{c}
\sum_{l=0}^{L-1} \hat{\hat{\mathbf{h}}}_{l}(k) q(k+l) \\
\downarrow \hat{\overline{\mathbf{v}}}(k-1)
\end{array}\right]
$$

This vector is the estimate of the mean $\overline{\mathbf{v}}(k)=$ $\sum_{l} \mathbf{h}(l) p(k-l)$. The shifting property of the signal and channel vectors has been exploited here to achieve computational efficiency.

4) Form the estimate of $\mathbf{v}_{0}(k)$ as

$$
\hat{\mathbf{v}}_{0}(k)=\mathbf{v}(k)-(\hat{\overline{\mathbf{v}}}(k)-\hat{\mathbf{h}}[k] p(k))
$$

5) Form the chip estimate as in (14).

6) Update the channel estimate:

$$
\hat{\mathbf{h}}[k+1]=\lambda \hat{\mathbf{h}}[k]+(1-\lambda) \hat{\mathbf{v}}_{0}(k) p^{*}(k)
$$

7) Truncate the channel estimate if $k>k_{\text {trunc. }}$. This is an important step in the algorithm, which ensures that only the significant channel coefficients will be used to filter the signal. The significant coefficients are those whose magnitudes are greater than $G \cdot h_{\max }$, where $h_{\max }$ is the maximum absolute value of the coefficients of the current channel estimate, and $G$ is a pre-specified truncation threshold. The rest of the channel estimate coefficients $\hat{\mathbf{h}}[k]$ are set to zero.

8) Update the carrier phase as in (22). Either of the two phase-error signals can be used.

9) Perform despreading as in (15).

10) Test for acquisition. If $N_{\text {test }}$ consequtive bit decisions at the despreader output have been equal to +1 , declare acquisition. Otherwise, continue the algorithm.

11) Once acquisition has been declared, determine the position $m$ of the channel estimate coefficient with the largest magnitude (coefficients are indexed from 0 (bottom)to $2 L-1$ (top)). The time-shifted received signal $v\left(k T_{c}+m T_{c} / 2\right)$ is synchronized with the sequence $p(k)$.

The algorithm is initialized with $\hat{\mathbf{h}}[0]=\mathbf{0}, \hat{\theta}(0)=0$.

The algorithm parameters are the the following:

- channel estimate length $L$

- despreader length $N$

- truncation threshold $G$

- time after which to begin truncation $k_{\text {trunc }}$

- filtering constants $\lambda$ and $K_{f 1,2}$

- acquisition test threshold $N_{\text {test }}$ (or other).

\section{PERFORMANCE RESULTS}

The proposed acquisition algorithm was tested in simulation. The spreading code is a length 63 Kasami sequence. The transmitter pulse is rectangular, of duration $T_{c}=0.2 \mathrm{~ms}$, corresponding to the chip rate of 4000 chips/sec. The channel has 3 paths, with total multipath spread of 26 chips or $6.5 \mathrm{~ms}$.

The system performance is first discussed for a timeinvariant, single-user channel. The channel coefficients have relative amplitudes $1,0.75$ and 0.5 , with corresponding delays of 0,4 and 26 chips. Geometric modeling of shallow water multipath would result in similar numbers, as discussed in [8]. Fig.1 illustrates the system performance on this channel, with additive white Gaussian noise at per-chip SNR of $-10 \mathrm{~dB}$. The starting time of the spreading code is chosen randomly. The starting time in this trial was 30. Shown in the figure on the left-hand side are the channel and its estimate. On the righthand side are the running despreader output, the corresponding bit decision made for the acquisition test, and the starting time estimate $m(k)$ obtained in each iteration. The system operation is shown for an entire block of 100 data bits.

Clearly, the estimated channel is a good match for the true one, and the position of largest magnitude coefficient determines the correct starting time of the code. The channel estimator uses $\lambda=0.999$, and truncation is performed after $k_{\text {trunc }}=126$ chips using truncation threshold $G=0.1$. Although the channel estimate spans 63 ships, only a few are kept. The running despreader length is $N=63$ chips. Bit estimates at its output are close to the ideal value +1 . Bit 
decisions made on the running despreader output indicate a +1 check already after 68 iterations. At this time, the correct starting time has not yet been acquired. The estimated starting time of the code, determined from the position of the largest channel estimate coefficient, is shown on the same scale below the despreader check function. The correct value, 30, is obtained in this trial after 163 chips, and acquisition is maintained thereafter. (These numbers provide a sense of how long the acquisition test should proceed before the acquisition decision is reached.)
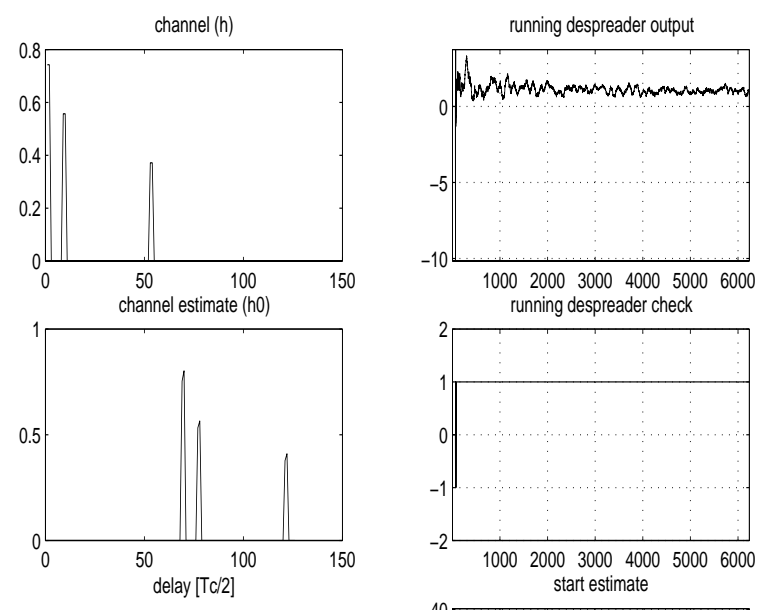

SNR=-10dB, L=63 chips/bit

sequence start=30; estimate $=30$

lambda $=0.999$

truncation threshold $=0.1$ (del.126)

despread length $=63$

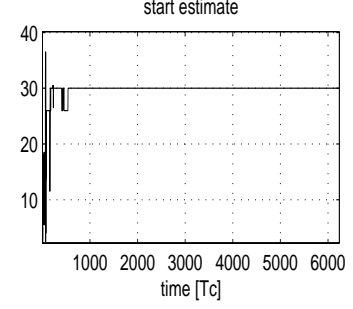

Fig. 1. Performance of the acquisition algorithm on the time-invariant AWGN channel.

One may wonder about the performance of the channel estimation algorithm (6) which operates directly on the received signal without subtraction of the inter-chip interference terms. The performance of this algorithm on the same channel is illustrated in Fig.2. The channel estimate is obviously inferior to that obtained using the principle (12). Degradation in the channel estimate is reflected in the poorer quality of bit estimates at the despreader output, which causes erroneous operation in the acquisition test and ultimately prolongs the acquisition time.

Acquisition time is defined here as the time it takes the acquisition algorithm to produce the correct estimate of the code timing, and maintain it for a certain number of chip intervals. Acquisition time is a random variable, and the performance of an acquisition scheme is judged by its mean value. Fig.3 shows the mean acquisition time as a function of SNR. Each value is a result of averaging over a number of simulation runs. In each run, acquisition is considered successful if correct timing estimate has been obtained and held for 1000 chips. If acquisition has not been acieved after $N_{\text {tot }}$ chip intervals, the
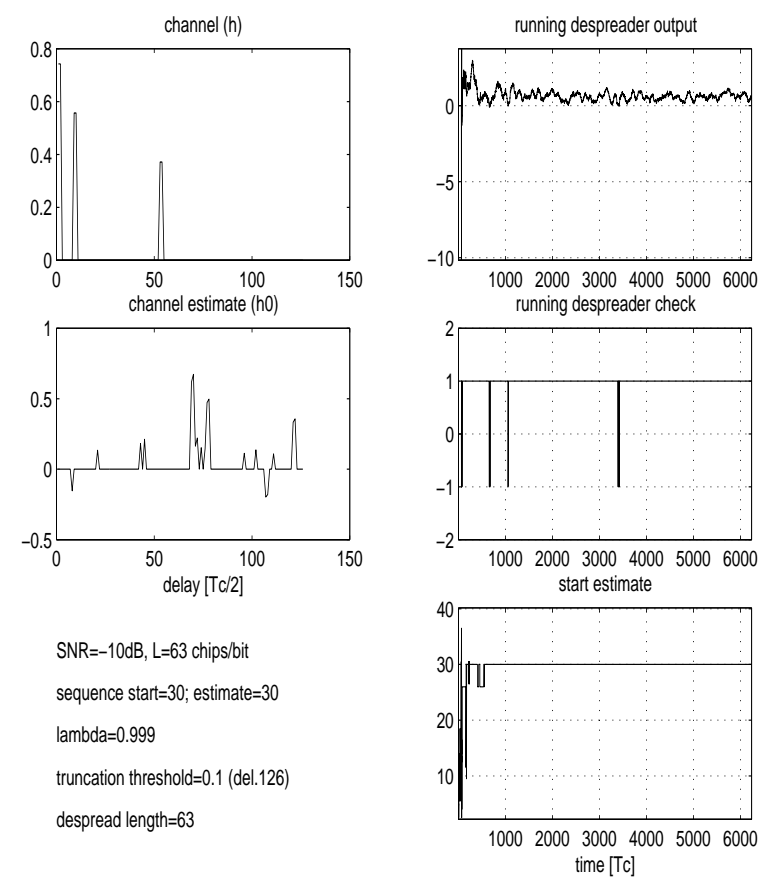

Fig. 2. Performance of the acquisition algorithm on the time-invariant AWGN channel, when channel estimation is performed without subtraction of interchip interference terms.

run is terminated. $N_{t o t}$ is chosen large enough that $90 \%$ or more trials result in acquisition. The mean acquisition time, obtained for a given SNR, is used in the system design to determine the interval allocated to the acquisition phase, i.e. the length of the all-ones sequence that must be transmitted before actual information transmission can begin.

Acquisition failure may occur either because the decision cannot be reached during the allocated time, or because a wrong decision is reached. The first type of acquisition error is controlled by selecting a long enough acquisition interval. The mean acquisition time for a given SNR is used to guide this selection. The second type of error is controlled by carefully designing the acquisition test, i.e. by selecting the despreader length $N$ and the test threshold $N_{\text {test }} . N$ is selected to provide enough processing gain that the SNR at despreader output is sufficiently high, say more than $5 \mathrm{~dB}$. Hence, a rule of thumb is $10 \log (N \cdot S N R)>5$. This selection ensures that bit decisions at despreader output are sufficiently reliable to guide the acquisition decision. $N_{\text {test }}$ should be selected large enough to ensure correlation between the despreader check function and the actual code acquisition. (If it is too small, false acquisition may occur.) To assist in selection of $N_{\text {test }}$, the dashed curve in Fig. 3 may be used. It represents the average number of chip intervals that elapse before the bit decision at the despreader output checks to +1 . Hence, the difference between the mean time to acquire and the mean time to check may serve to guide the selection of the acquisition test length.

System performance on a time-varying channel and in the 


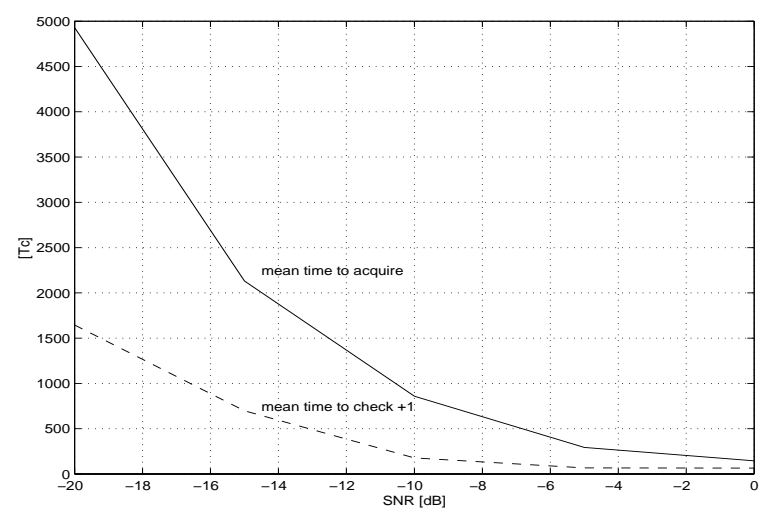

Fig. 3. Mean acquisition time for the 3-path time-invariant channel model.

presence of multiuser interference is studied next. Fig.4 illustrates the results of a four-user trial. Each of the four signals passes through an independently fading channel. Each channel is modeled as having 3 paths, with relative mean path gains $1,0.75$, and 0.5 , and different path delays, randomly generated within the overall multipath spread of 26 chips. The path gains are time-varying, complex-valued independent random processes, generated using a second-order auto-regressive model driven by a zero-mean white Gaussian process. The 3$\mathrm{dB}$ bandwidth of the Doppler power spectrum of each channel tap process defines its Doppler spread. In addition, each user is assumed to move at a different velocity $v$, resulting in the Doppler shift $f_{d}= \pm(v / c) f_{c}$. The value $c=1500 \mathrm{~m} / \mathrm{s}$ for the speed of sound, and $f_{c}=15 \mathrm{kHz}$ for the carrier frequency were used. The velocities were randomly chosen between 0 and 5 knots. The users are assigned spreading codes from the length 63 Kasami set (there are 8 codes in this set), with each users' code having a randomly selected starting phase. The signals arrive at the receiver asynchronously. The power of each interferer is equal to the power of the desired signal, resulting in the signal-to-interference ratio $\mathrm{SIR}=-5 \mathrm{~dB}$.

Shown in Fig.4, on the right-hand side, are the three pathgain processes that describe the desired user's channel. The Doppler spread, chosen equal for all paths, is $1.2 \mathrm{~Hz}$, which results in the normalized value $\omega_{d} T_{c}=10^{-3}$. This value is at the limit of coherent systems' capabilities, and higher than what is found in many applications. It was chosen to demonstrate the system robustness in extreme conditions of fast fading. The performance is again shown for an extended period of time (longer than needed to acquire the timing). The phase plot on the left-hand side shows the true and the estimated phase. The desired user moves at 2 knots, and the resulting Doppler shift of $10 \mathrm{~Hz}$ is accurately tracked by the PLL. The first form of the phase error signal (21) is used in this example. The second form gives equally good results. It is worth mentioning that the presence of different Doppler shifts among the users actually helps the overall channel estimation process. The residual Doppler shift in the interfering users' signals causes the composite interference to appear less coherent (more noise-like).
When there is no frequency offset, the interference suppression capability is limited by the (non-zero) cross-correlations of the spreading codes. The per-bit SNR in this example is 20 $\mathrm{dB}$, i.e., the per-chip SNR at the receiver input is $2 \mathrm{~dB}$ for the code length of 63 . The receiver parameters are indicated in the figure. The decisions made at the despreader output produce $\mathrm{a}+1$ check soon after initialization, and the code timing is acquired thereafter. It is very interesting to observe the estimated timing in the last plot. The starting time of the sequence is 30 , and the acquisition algorithm reaches this value after about 900 chip intervals. If the channel were time-invariant, this value would be maintained throughout the observation period. However, because the channel varies in time, the relative strength of the path gains changes. Consequently, the path which was originally the strongest one may become a secondary path at some point. This is exactly the case in the example shown. Careful observation of the channel tap gains shows that the first path is not the strongest at all times. The acquisition algorithm follows these changes accurately, as demonstrated by the period of time when timing estimate deviates from the value 30 , only to emerge again later as the first path regains strength. The speed and accuracy of tracking are influenced by the choice of $\lambda$ and $G$, whose optimal values depend on the noise level and the rate of channel variation. At the moment, these values are determined heuristically.
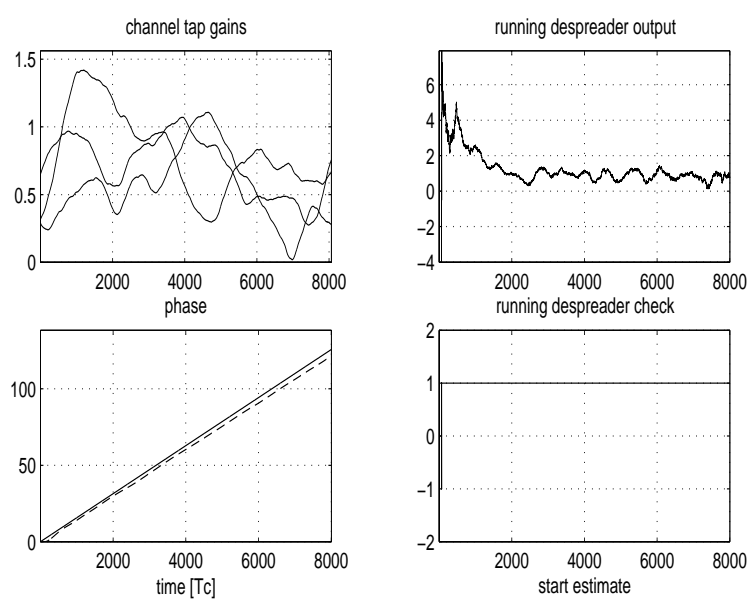

$\mathrm{SIR}=-5 \mathrm{~dB}$, wdTc=10e-4

$\mathrm{SNRb}=20 \mathrm{~dB}, \mathrm{~L}=63 \mathrm{chips} / \mathrm{bit}$

sequence start=30; estimate $=30$

lambda $=0.999, \mathrm{Kf} 1=0.0001, \mathrm{Kf2}=\mathrm{Kf} 1 / 10$

truncation threshold $=0.25$ (del.126)

despread length $=63$

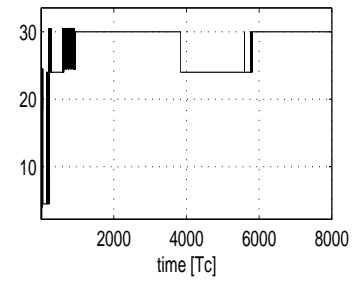

Fig. 4. Performance of the acquisition algorithm on a time-varying channel with four users. Each user's channel is an independent 3-path Rayleigh fading channel. Users move at different velocities (few m/s). Kasami sequences with randomly selected starting phase are used. The signals are of equal power and asynchronous. 


\section{CONCLUSION}

Code acquisition in DS/SS systems operating over frequency-selective time-varying underwater acoustic channels was investigated. The problem of acquisition was cast into the framework of channel estimation. An adaptive channel estimator, based on a computationally efficient procedure of inter-chip interference removal and tap-selective updating was proposed. System performance was demonstrated through numerical examples, showing excellent results in both time-invariant and time-varying channels, in low-SNR or multiple-access regime.

Future work in this area should focus on both experimental and analytical results. Experimental demonstration of the algorithm proposed should be conducted using real data collected in different underwater communication scenarios involving multiple autonomous vehicles. Analytical work should address theoretical assessment of acquisition performance and selection of optimal receiver parameters, as well as extension of acquisition principles to multiuser case (when multiple signals are acquired simultaneously) and to multichannel receivers.

\section{REFERENCES}

[1] L.Freitag, M.Stojanovic, S.Singh and M.Johnson, "Analysis of channel effects on direct-sequence and frequency-hopped spread-spectrum acoustic communication," IEEE Journal of Oceanic Engineering, vol.26, No.11, Oct. 2001, pp.586-593.

[2] J.K.Holmes, Coherent Spread Spectrum Systems, Malabar, FL: Krieger Publishing Company, 1990.

[3] J.G.Proakis, Digital Communications, New York: Mc-Graw Hill, 1995.

[4] M.G.El-Tarhuni and A.U.H.Sheikh, "Code acquisition of DS/SS signals in fading channels using an LMS adaptive filter," IEEE Trans. Commun., vol.2, No.4, Apr.1998, pp.85-88.

[5] U.Madhow, "MMSE interference suppression for timing acquisition and demodulation in direct-sequence CDMA systems," IEEE Trans. Commun., pp.1065-1075, Aug. 1998.

[6] R.Smith and S.L.Miller, "Acquisition performance of an adaptive receiver for DS-CDMA," IEEE Trans. Commun., vol.47, No.9, Sept. 1999, pp.1416-1424.

[7] M.Stojanovic and L.Freitag, "Multiuser undersea acoustic communications in the presence of multipath propagation," in Proc. IEEE Oceans'01 Conference, Honolulu, HI, November 2001.

[8] M.Stojanovic and L.Freitag, "Hypothesis-feedback equalization for direct-sequence spread-spectrum underwater communications," in Proc. IEEE Oceans'00 Conference, Sept. 2000.

[9] M.Stojanovic, L.Freitag and M.Johnson, "Channel-estimation-based adaptive equalization of underwater acoustic signals,"in Proc. IEEE Oceans'99 Conference, Seattle, WA, September 1999.

\section{ACKNOWLEDGMENT}

This work is part of the project "Very Shallow Water Surf Zone Mine Countermeasures" (VSW/SZ MCM), supported by ONR. 